\title{
Improvement and Aggravation of Spontaneous Unruptured Vertebral Artery Dissection
}

\author{
Tomoya Shibahara $^{a}$ Masahiro Yasaka ${ }^{a}$ Yoshiyuki Wakugawa ${ }^{a}$ \\ Koichiro Maeda ${ }^{a}$ Takeshi Uwatoko ${ }^{a}$ Takahiro Kuwashiro $^{a}$ \\ Gregory Y.H. Lip ${ }^{b}$ Yasushi Okada ${ }^{a}$ \\ a Department of Cerebrovascular Medicine and Neurology, Clinical Research Institute,

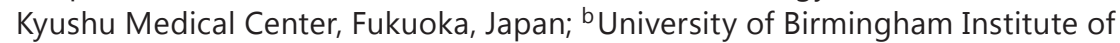 \\ Cardiovascular Sciences, City Hospital, Birmingham, UK
}

\section{Keywords}

Vertebral artery dissection · Vascular imaging · Asian ethnicity · Risk factor · Smoking

\begin{abstract}
Background: Intracranial vertebral artery dissection (VAD) is a well-recognized cause of stroke in young and middle-aged individuals, especially in Asian populations. However, a long-term natural course remains unclear. We investigated the long-term time course of VAD using imaging findings to examine the rate and predisposing factors for improvement. Methods: We registered 56 consecutive patients (40 males; mean age, $51.8 \pm 10.7$ years) with acute spontaneous VAD and retrospectively investigated neuroimaging and clinical course within 1 month and at 3 months \pm 2 weeks, 6 months \pm 2 weeks, and 12 months \pm 2 weeks after onset to ascertain predisposing factors and time course for improvement. Results: The most common presenting symptoms were headache and/or posterior neck pain, seen in 41 patients (73\%). Magnetic resonance imaging showed brainstem and/or cerebellum infarction in only 32 patients (57\%). Of the 56 VADs, 16 (28\%) presented with pearl and string sign, 5 (9\%) with pearl sign, 15 (27\%) with string sign, and 20 (36\%) with occlusion sign. VAD occurred on the dominant side in 20 patients and on the nondominant side in the other 36 patients. The pearl and string sign was more frequently noted on the dominant side than on the nondominant side ( 50 vs. $17 \%, p=0.008$ ). On the other hand, occlusion occurred more often on the nondominant side than on the dominant side ( 47 vs. $15 \%, p=0.016)$. Furthermore, the pearl and string sign was more frequently seen in the improvement group ( $41 \mathrm{vs.} 15 \%, p=0.028$ ), whereas the occlusion sign was evident more frequently in the nonimprovement group ( $21 \mathrm{vs.} 52 \%$,
\end{abstract}


$p=0.015)$. Follow-up neuroimaging evaluation was performed at 1 and 3 months in $91 \%$ each, and at 6 and 12 months in $82 \%$ each. VAD aggravation was identified within 1 month after onset in $14 \%$, while VAD improvement was seen in $14,38,50$, and $52 \%$ at each period, mainly within 6 months after onset. Older patients and current smoking were negatively associated with VAD improvement. Conclusions: VAD improvement primarily occurs within 6 months after onset, and VAD aggravation within 1 month. It seems that older patients and current smoking are negative predictors of VAD improvement as risk factors, and as image findings, the pearl and string sign is a positive predictor and occlusion a negative predictor.

(c) 2017 The Author(s)

Published by S. Karger AG, Basel

\section{Introduction}

Cervical artery dissection accounts for only $1-2 \%$ of all ischemic strokes, but it accounts for $10-25 \%$ of strokes in young and middle-aged individuals [1]. Ethnic differences are seen in the site of dissection. Although extracranial and internal carotid artery dissections are common in Caucasian populations, intracranial vertebral artery dissection (VAD) is more common in Asian populations [2]. Minor or trivial trauma, sports activities, daily activities, and potentially even excessive torsion of the cervical spine may sometimes cause VAD. Hypertension, diabetes mellitus, hypercholesterolemia, smoking, and obesity are also well known as important risk factors for vascular disease; however, whether such factors are associated with VAD remains unclear.

Although digital subtraction angiography (DSA) has been the gold standard for the diagnosis and follow-up of VAD, noninvasive methods such as magnetic resonance angiography (MRA) and computed tomography angiography (CTA) have recently proven useful for the detection of VAD [3-5]. However, MRA studies of the long-term natural course of VAD are limited. For example, Arauz et al. [6] studied the predictors and temporal course of recanalization after VAD in Mexico City and reported that recanalization of VAD occurred mainly within 6 months after symptom onset, with no significant association between clinical factors and recanalization. Although this result may be helpful in deciding on discontinuation of antithrombotic therapy and appropriate follow-up examinations for neuroimaging, whether this result is applicable to East Asian populations has yet to be established, given the ethnic differences in VAD mentioned above.

In this study, we investigated the long-term time course of VAD using imaging findings to examine the rate and predisposing factors for improvement in a consecutive cohort of Japanese patients with spontaneous VAD.

\section{Methods}

\section{Patients}

This study was a retrospective, single-center study in the Department of Cerebrovascular Medicine and Neurology, Clinical Research Institute, Kyushu Medical Center. We registered 56 consecutive patients (40 males; mean age, $51.8 \pm 10.7$ years) with acute spontaneous VAD who had been admitted to our hospital between November 2002 and March 2013. All patients had undergone a neurological examination that included assessment of ischemic or hemorrhagic lesions and bilateral vertebral arteries. All patients had received magnetic resonance imaging (MRI) including DWI and MRA, and one or both vascular imaging studies including DSA and CTA within 1 month after onset. We defined the onset of clinical symptoms on the basis of the first symptom, which the stroke neurologist could associate with VAD. All patients had met the following inclusion criteria: (a) a history of acute clinical symptoms and/or signs relevant to VAD, (b) vascular imaging evidence of VAD (i.e., pearl and string sign, aneurysmal dilatation, string sign, or tapered or abrupt occlusion). Exclusion criteria were as follows: (a) definitively traumatic VAD secondary to major trauma, (b) 
cases of subarachnoid hemorrhage caused by VAD, (c) incidentally found asymptomatic fusiform dilatations of VA, or (d) laboratory or vascular imaging findings suggestive of vasculitis or fibromuscular dysplasia. In this study, spontaneous VAD was defined as occurring spontaneously, after physical exertion, or after a so-called minor trauma such as sports activity or cervical manipulative therapy [7, 8].

\section{Initial and Follow-Up Radiological Evaluation}

The primary vascular imaging shapes of VADs were classified according to morphological lesion type as follows: (1) pearl and string sign, as stenosis involving irregular long or short segments; (2) pearl sign, as pure aneurysmal dilatation of the vertebral artery (VA); (3) string sign, as irregular or smooth stenosis of the VA extending a variable distance; and (4) occlusion sign, as gradual tapering or abrupt occlusion of the VA involving either the entire artery or only one segment of the artery $[9,10]$. All images were reviewed by at least one experienced neuroradiologist and the primary stroke neurologist in charge. In evaluating the VAs, differentiating a dissected VA from an atherosclerotic VA is important. In this study, atherosclerosis of the VAs was diagnosed if luminal irregularities, stenosis, or occlusions were found not only in the VAs, but also in the other cervical and intracranial arteries. We judged ischemic stroke caused by VAD based on both neurological symptoms and diffusion-weighted imaging.

The location of dissection was classified as V1, V2, V3, or V4. V1 was the segment of the VA that passes into the neck and enters the transverse foramen of C6. V2 was the segment that ascends through the transverse foramen from C6 to C2. V3 exited the transverse foramen of C2, wound around C1 posteriorly in a tortuous manner, and entered the dura at the foramen magnum. V4 began at the foramen magnum and united with the contralateral VA to form the basilar artery. To determine the dominant side of VA, we collected the diameter of the bilateral VAs at the V2 segment measured by carotid ultrasonography at admission in all cases, in addition to DSA, MRA, or CTA. We compared the diameters of bilateral VAs and judged the VA with a diameter wider than the contralateral side as the dominant side, and that with a diameter the same as or smaller to be the nondominant side.

Initial neuroradiological studies including neuroimaging were performed on admission. We retrospectively investigated both the rate of follow-up evaluation and the rate of VAD improvement within 1 month and at 3 months \pm 2 weeks, 6 months \pm 2 weeks, and 12 months \pm 2 weeks after onset. We evaluated VAD improvement by using follow-up vascular imaging. Improvement of VAD was defined as recanalization, reduction in the degree of stenosis, or reduction of aneurysm. Aggravation of VAD was defined as any progression of stenosis, any aneurysmal enlargement, or new appearance of an aneurysm. We regarded a patient as showing no improvement when any deterioration of vascular lesions was evident, surgical treatment was performed, or the patient died during follow-up. Even in cases with VAD aggravation, we only judged improvement as present when the VAD had improved compared with the initial findings after aggravation. Improvement and aggravation on follow-up examination were evaluated with the same modalities (DSA, CTA, or MRA) as the initial examination. We assessed functional outcome at discharge according to the medical records using the modified Rankin Scale, with scores of 0-1 defined as a "favorable" outcome, and scores of 2-6 defined as "unfavorable." We evaluated the symptomatic stroke/TIA recurrence rate during the follow-up by investigating new ischemic symptoms in the medical record and/or new ischemic infarction in the MRI.

\section{Variable Definitions}

The following characteristics derived from patient interviews or medical records were assessed at baseline: current smoker, had smoked $>100$ cigarettes in their lifetime and had smoked in the last 28 days; hypertension, history of elevated blood pressure (systolic blood pressure $\geq 140 \mathrm{~mm} \mathrm{Hg}$ or diastolic blood pressure $\geq 90 \mathrm{~mm} \mathrm{Hg}$ ) diagnosed by the treating physician or use of antihypertensive treatment; diabetes mellitus, history of diabetes mellitus diagnosed by the treating physician with fasting glucose $>7 \mathrm{mmol} / \mathrm{L}$ or hemoglobin $\mathrm{A}_{1 \mathrm{c}}$ (National Glycohemoglobin Standardization Program [NGSP]) on admission $\geq 6.5 \%$, or use of an antidiabetic treatment; dyslipidemia, total cholesterol $>5.69 \mathrm{mmol} / \mathrm{L}$, low-density lipoprotein cholesterol $>3.62 \mathrm{mmol} / \mathrm{L}$, high-density lipoprotein cholesterol $>1.03 \mathrm{mmol} / \mathrm{L}$, or triglyceride $>1.69 \mathrm{mmol} / \mathrm{L}$ on admission or diagnosed by the treating physician, or use of an antidyslipidemic treatment. We also investigated the use of any antithrombotic therapy (antiplatelet or anticoagulant, or both) after hospitalization.

Statistical Analyses

Statistical analyses were performed using JMP software, version 10 (SAS Institute, Cary, NC, USA). To compare clinical, physiological, and radiological factors, we divided patients into 2 groups: with and without 


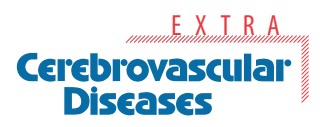

Table 1. Initial symptoms due to vertebral artery dissection in 56 patients

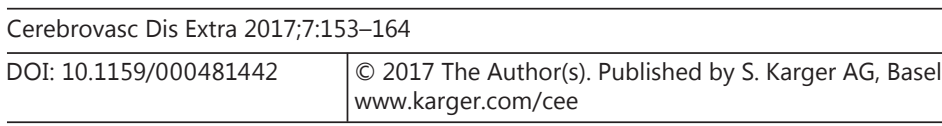

Shibahara et al.: Improvement of Vertebral Artery Dissection

\begin{tabular}{lc}
\hline Symptom & Patients, $n(\%)$ \\
\hline Headache and/or posterior neck pain & $41(73)$ \\
Vertigo & $12(21)$ \\
Dizziness & $12(21)$ \\
Nausea, vomiting & $11(20)$ \\
Dysesthesia & $8(14)$ \\
Dysarthria, dysphagia & $3(5)$ \\
Hemiparesis & $1(2)$ \\
Hearing loss & $1(2)$ \\
\hline
\end{tabular}

improvement of VAD at 12 months. Cohen's kappa was calculated to evaluate interobserver agreement. The value was interpreted according to Landis and Koch [11] with a value of 0 as poor, $0.01-0.20$ slight, $0.21-0.40$ fair, 0.41-0.60 moderate, $0.61-0.80$ substantial, and 0.81-1.0 almost perfect agreement. The $\chi^{2}$ test, unpaired $t$ test, Wilcoxon signed-rank sum test, or McNemar test were performed as appropriate. The following variables were analyzed: age, sex, hypertension, diabetes mellitus, dyslipidemia, current smoking, alcohol use, minor trauma, baseline National Institutes of Health Stroke Scale score, ischemic stroke, dominant side of VAD, antiplatelet treatment, anticoagulant treatment, favorable outcome, and vascular imaging. Logistic regression analysis with a forward stepwise method was then performed to identify any independent association of improvements in VAD with other clinical, physiological, and radiological factors. The cutoff in the univariate analyses as required for inclusion in multivariable analyses was $p<0.25$.

Ethical Considerations

This study was conducted with the approval of the Kyushu Medical Center Institutional Review Board for Clinical Research.

\section{Results}

\section{Clinical Features}

Initial symptoms of the 56 patients are listed in Table 1 . The most common presenting symptoms were headache and/or posterior neck pain, seen in 41 patients (73\%), followed by vertigo (21\%), dizziness (21\%), and nausea/vomiting (20\%).

MRI showed brainstem and/or cerebellum infarction in only 32 patients (57\%). Symptomatic ischemic stroke was seen in 25 patients (44\%) and asymptomatic ischemic stroke with only headache and/or posterior neck pain in 7 patients $(13 \%)$. Wallenberg syndrome was seen in 16 patients (28\%), all of whom had lateral medullary infarction. Possible dissection-related minor trauma was found in 18 patients (32\%), arising during activities such as golf, baseball, abdominal exercise, stretching of the neck, chiropractic manipulations, and coughing.

\section{Vascular Lesions at First Examination}

Of the 56 VADs, MRI/MRA was performed at initial diagnosis in all patients. DSA was examined in 53 and CTA in 8 patients, respectively (see online suppl. Table I, for all online suppl. material, see www.karger.com/doi/10.1159/000481442). The median interval from the onset to initial MRA was 6 days (range, 1-31 days). We found 53 VADs (96\%) at the V4 segment, $3(5 \%)$ at the V1-2 segment, and $1(2 \%)$ at the V3-4 segment. The pearl and string sign was seen in 16 patients (28\%), pearl sign in 5 (9\%), string sign in $15(27 \%)$, and occlusion sign in $20(36 \%)$. VAD occurred on the dominant side in 20 patients and on the nondominant side in the other 36 patients (Table 2 ). The pearl and string sign was more frequently noted 
Table 2. Primary vascular imaging of the dominant/nondominant side

\begin{tabular}{lcccc}
\hline Primary vascular imaging & $\begin{array}{l}\text { All, } \\
n(\%)\end{array}$ & $\begin{array}{l}\text { Dominant } \\
\text { side, } n(\%)\end{array}$ & $\begin{array}{l}\text { Nondominant } \\
\text { side, } n(\%)\end{array}$ & $p$ \\
\hline Pearl and string & $16(28)$ & $10(50)$ & $6(17)$ & 0.008 \\
Pearl & $5(9)$ & $3(15)$ & $2(6)$ & 0.235 \\
String & $15(27)$ & $4(20)$ & $11(30)$ & 0.393 \\
Occlusion & $20(36)$ & $3(15)$ & $17(47)$ & 0.016 \\
\hline Total & $56(100)$ & $20(100)$ & $36(100)$ & \\
\hline
\end{tabular}

Fig. 1. The proportion and time course of achieving vertebral artery dissection (VAD) improvement at $1,3,6$, and 12 months of follow-up is shown. Improvement mainly occurred up to 6 months after onset. $* p<0.05$, McNemar test.

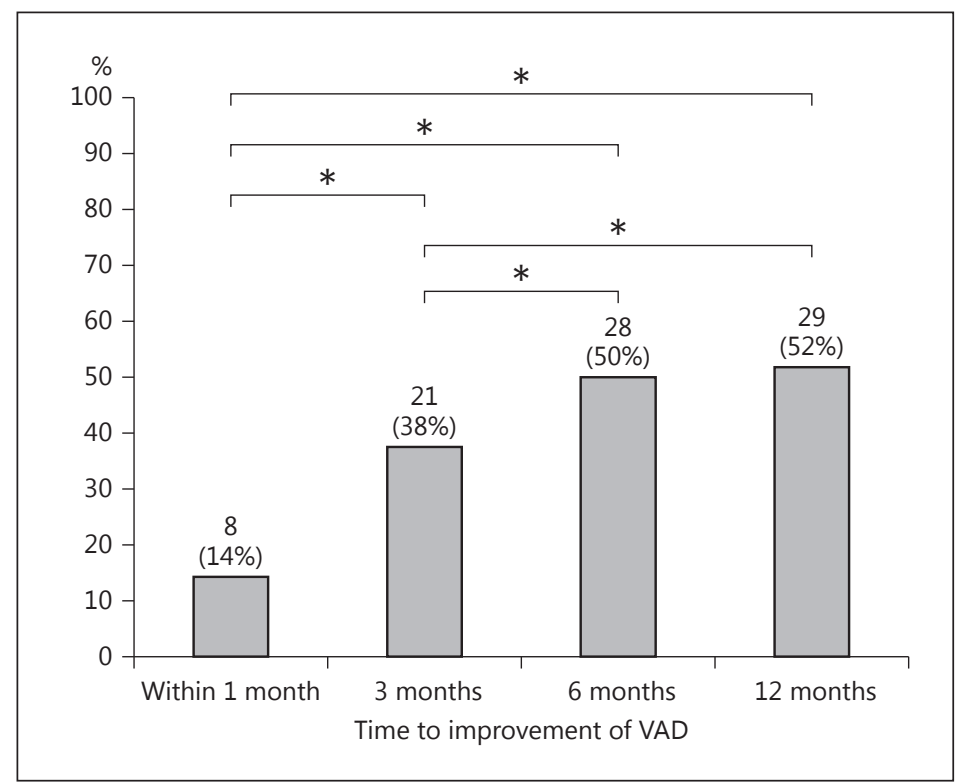

on the dominant side than on the nondominant side (50 vs. $17 \%, p=0.008$ ). On the other hand, occlusion occurred more often on the nondominant side than on the dominant side (47 vs. $15 \%, p=0.016)$.

\section{Changes in Vascular Lesions on Follow-Up Examination}

Follow-up vascular imaging was performed in 51 patients (91\%) within 1 month, 51 patients (91\%) at 3 months, 46 patients (82\%) at 6 months, and 46 patients (82\%) at 12 months (see online suppl. Table I). Among the 53 patients (95\%) who underwent DSA on admission, we compared the DSA at each period with the first one. If initial or follow-up DSA was not performed, we evaluated in the same way using MRA or CTA. Of the 29 cases of VAD improvement, 10 were evaluated with DSA, 2 with CTA, and 17 with MRA. The interobserver agreement measured by a Cohen's kappa value was 0.78 (95\% CI, 0.62-0.95), which is a substantial agreement. Improvement of VAD was confirmed in 8 patients (14\%) within 1 month, 21 patients (38\%) at 3 months, 28 patients (50\%) at 6 months, and 29 patients (52\%) at 12 months (Fig. 1). Of the 8 cases with VAD improvement within 1 month, 2 cases were within 7 days, 3 cases were 8-14 days, and 3 cases were 15-21 days. A trend toward VAD improvement was seen up to 6 months after onset. 

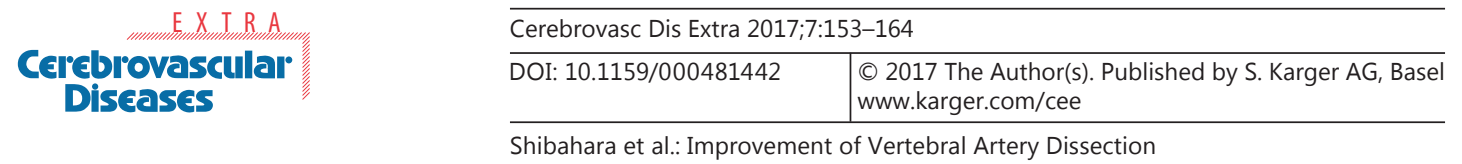

Table 3. Pattern of vertebral artery dissection (VAD) improvement at 12 months, ischemic stroke associated with VAD, and functional outcome at discharge by primary vascular imaging

a Pattern of VAD improvement at 12 months

\begin{tabular}{|c|c|c|c|c|c|c|c|c|}
\hline \multirow{2}{*}{$\begin{array}{l}\text { Primary vascular } \\
\text { imaging }\end{array}$} & \multirow[t]{2}{*}{ Total, $n$} & \multicolumn{4}{|c|}{ Pattern of improvement, $n$} & \multicolumn{2}{|c|}{ Improvement, $n(\%)$} & \multirow[t]{2}{*}{$p$} \\
\hline & & $\begin{array}{l}\text { recana- } \\
\text { lization }\end{array}$ & $\begin{array}{l}\text { reduction } \\
\text { of stenosis }\end{array}$ & $\begin{array}{l}\text { reduction of } \\
\text { aneurysm }\end{array}$ & $\begin{array}{l}\text { reduction of } \\
\text { stenosis and } \\
\text { aneurysm }\end{array}$ & $\begin{array}{l}\text { yes } \\
(n=29)\end{array}$ & $\begin{array}{l}\text { no } \\
(n=27)\end{array}$ & \\
\hline Pearl and string & 16 & 0 & 5 & 0 & 7 & $12(41)$ & $4(15)$ & 0.028 \\
\hline Pearl & 5 & 0 & 0 & 1 & 0 & $1(3)$ & $4(15)$ & 0.136 \\
\hline String & 15 & 0 & 10 & 0 & 0 & $10(35)$ & $5(18)$ & 0.178 \\
\hline Occlusion & 20 & 6 & 0 & 0 & 0 & $6(21)$ & $14(52)$ & 0.015 \\
\hline
\end{tabular}

b Ischemic stroke associated with VAD and functional outcome at discharge

\begin{tabular}{|c|c|c|c|c|c|c|c|}
\hline \multirow[t]{2}{*}{$\begin{array}{l}\text { Primary } \\
\text { vascular imaging }\end{array}$} & \multirow[t]{2}{*}{$\begin{array}{l}\text { All, } \\
n(\%)\end{array}$} & \multicolumn{3}{|c|}{ Ischemic stroke associated with VAD } & \multicolumn{2}{|c|}{$\begin{array}{l}\text { Modified Rankin Scale at } \\
\text { discharge }\end{array}$} & \multirow[b]{2}{*}{$p$} \\
\hline & & $\begin{array}{l}\text { with, } \\
n(\%)\end{array}$ & $\begin{array}{l}\text { without, } \\
n(\%)\end{array}$ & $p$ & $\begin{array}{l}\text { favorable, } \\
n(\%)\end{array}$ & $\begin{array}{l}\text { unfavorable, } \\
n(\%)\end{array}$ & \\
\hline Pearl and string & $16(28)$ & $3(10)$ & $13(54)$ & $<0.001$ & $14(33)$ & $2(15)$ & 0.230 \\
\hline Pearl & $5(9)$ & $1(3)$ & 4 (17) & 0.079 & $4(9)$ & $1(8)$ & 0.858 \\
\hline String & $15(27)$ & $10(31)$ & $5(21)$ & 0.384 & $13(30)$ & $2(15)$ & 0.290 \\
\hline Occlusion & $20(36)$ & $18(56)$ & $2(8)$ & $<0.001$ & $12(28)$ & $8(62)$ & 0.027 \\
\hline Total & $56(100)$ & $32(100)$ & $24(100)$ & & $43(100)$ & $13(100)$ & \\
\hline
\end{tabular}

The primary modality of vascular imaging and the pattern of VAD improvement are listed in Table 3a. The pearl and string sign group showed improvement in 12 of 16 patients $(75 \%)$, and we present a representative example with improvement 3 months after onset (Fig. 2). Reductions in stenosis and aneurysm were seen in 7 patients, whereas reduction in stenosis only was seen in 5 patients. The string sign group showed reduction of stenosis in 10 of 15 patients $(67 \%)$. On the other hand, the pearl sign group revealed reduction of aneurysm in only 1 of 5 patients (20\%), and the occlusion sign group showed recanalization in only 6 of 20 patients $(30 \%)$.

Aggravation of lesions was shown in 8 patients (14\%), all of whom showed aggravation within 1 month of onset. An enlargement or new appearance of aneurysm was noted in 4 cases, with 3 cases receiving surgical or endovascular interventional treatment. Exacerbation of stenosis was confirmed in 4 patients. No association was seen between any factors and aggravation of VAD.

Ischemic stroke associated with VAD occurred less frequently in the pearl and string sign ( 10 vs. $54 \%, p<0.001)$, whereas it was seen more frequently in the occlusion sign ( 56 vs. $8 \%$, $p<0.001$ ) (Table 3b). Furthermore, an unfavorable outcome (modified Rankin Scale 2-6) at discharge was more common in the occlusion sign group (28 vs. 62\%, $p=0.027$ ). Of the 56 VADs, no patients developed symptomatic stroke/TIA recurrence.

\section{Predictors of VAD Improvement}

The presenting clinical characteristics of 29 VADs (52\%) with improvement and 27 VADs (48\%) without improvement during the 12 months of follow-up are shown in Table 

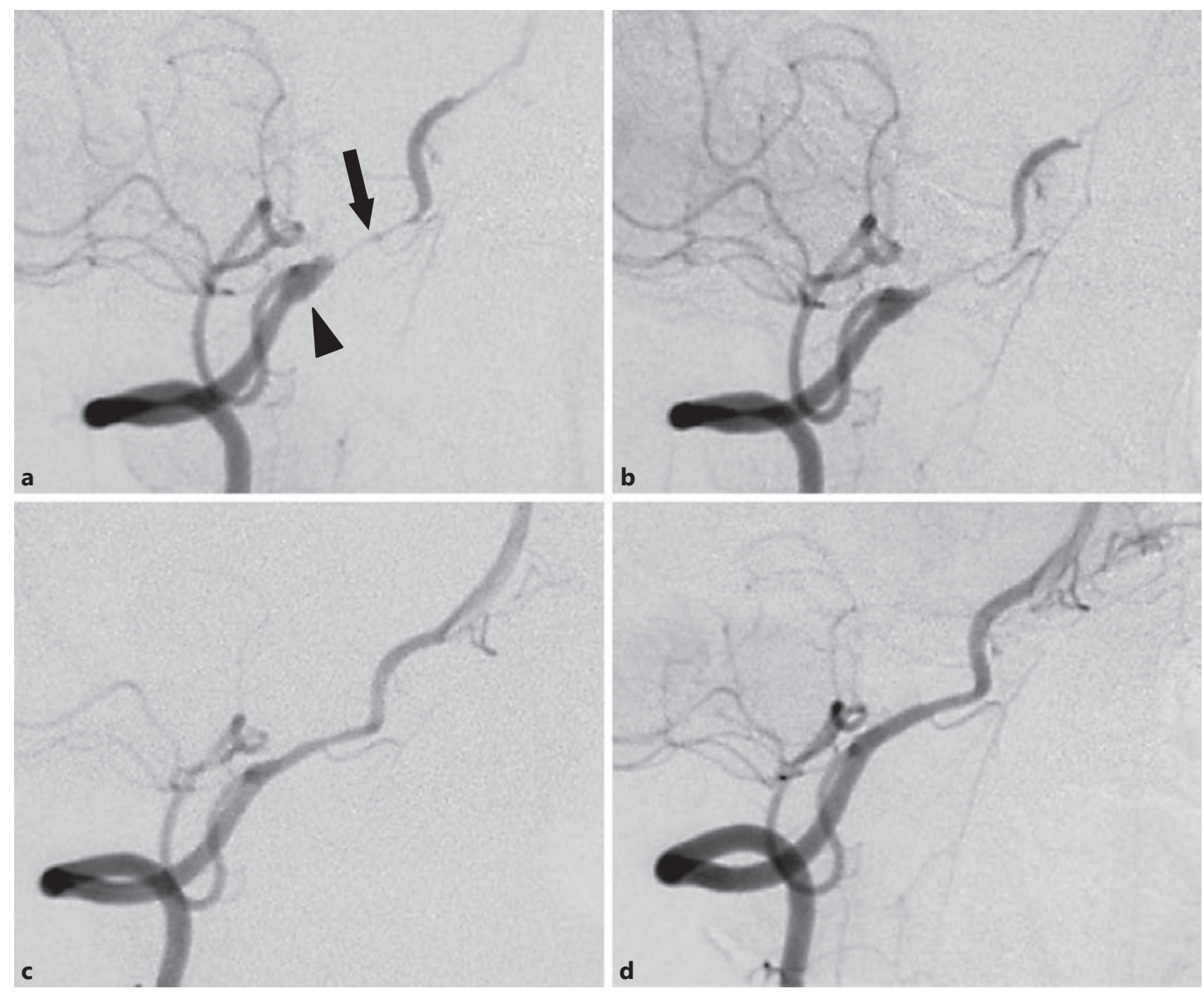

Fig. 2. Changes in the right vertebral arterial angiographic findings during 6 months in a 36-year-old man who developed vertebral artery dissection with sudden-onset right occipital pain. a Angiogram 10 days after the onset demonstrated pearl and string sign, with dilation (arrowhead) of the right vertebral artery at the branching of the posterior inferior cerebellar artery with distal stenosis (arrow). b On day 21, there was no significant change. $\mathbf{c}$ On day 91, both dilation and stenosis improved. $\mathbf{d}$ The 6-month follow-up angiogram showed almost normal morphology of the right vertebral artery.

4. A favorable outcome (modified Rankin Scale 0-1) at discharge was observed in 43 of the 56 patients $(77 \%)$ and was as frequent in VAD with improvement (23 of 29 VADs, 79\%) as in VAD without improvement (20 of 27 VADs, 74\%, $p=0.643$ ). Univariate analysis showed that older age and current smoking were more frequent in the VAD group without improvement at 12 months ( $p=0.043$ and $p=0.029$, respectively). On multivariable regression analysis, increasing age (odds ratio [OR], 1.07; 95\% confidence interval [CI], $1.00-1.15, p=0.045$ ) and current smoking (OR, 5.66; 95\% CI, 1.47-27.38, $p=0.010$ ) were independently associated with no improvement of VAD. Furthermore, the pearl and string sign was more frequently seen in the improvement group ( 41 vs. $15 \%, p=0.028$ ), whereas the occlusion sign was evident more frequently in the nonimprovement group ( $21 \mathrm{vs.} 52 \%$, $p=0.015$ ) (Table 3a).

Antithrombotic therapies in each period are listed in online supplementary Table II. Thirty-one patients (55\%) were treated with antithrombotic therapy. Anticoagulation therapy was mainly selected on admission, whereas antiplatelet therapy was frequently 

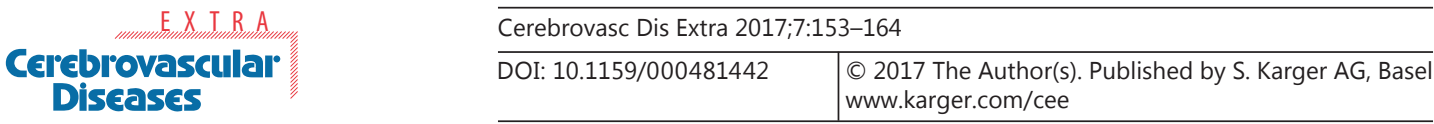

Shibahara et al.: Improvement of Vertebral Artery Dissection

Table 4. Baseline characteristics of the patients with and without improvement at 12 months

\begin{tabular}{|c|c|c|c|c|c|}
\hline \multirow[t]{2}{*}{ Baseline characteristics } & \multicolumn{2}{|c|}{ Improvement at 12 months } & \multirow[t]{2}{*}{$p^{*}$} & \multirow[t]{2}{*}{ OR $(95 \% \mathrm{CI})^{\dagger}$} & \multirow[t]{2}{*}{$p^{\dagger}$} \\
\hline & yes $(n=29)$ & no $(n=27)$ & & & \\
\hline Age, years & $49 \pm 9$ & $55 \pm 12$ & 0.043 & $1.07(1.00-1.15)$ & 0.045 \\
\hline Male & $18(62)$ & $22(81)$ & 0.108 & & \\
\hline Hypertension & $18(62)$ & $17(63)$ & 0.944 & & \\
\hline Diabetes mellitus & $2(7)$ & $4(15)$ & 0.338 & & \\
\hline Dyslipidemia & $15(52)$ & $13(48)$ & 0.789 & & \\
\hline Current smoking & $12(41)$ & $19(70)$ & 0.029 & $5.66(1.47-27.38)$ & 0.010 \\
\hline Alcohol use & $16(55)$ & $18(67)$ & 0.379 & & \\
\hline Minor trauma & $10(34)$ & $8(30)$ & 0.698 & & \\
\hline NIHSS & $0(0-2)$ & $1(0-2)$ & 0.216 & & \\
\hline Ischemic stroke & $14(48)$ & $18(67)$ & 0.164 & & \\
\hline Intracranial VAD & $26(90)$ & $26(96)$ & 0.335 & & \\
\hline Dominant side of VAD & $10(35)$ & $8(30)$ & 0.698 & & \\
\hline Antiplatelet & $15(52)$ & $13(49)$ & 0.789 & & \\
\hline Anticoagulant & $12(41)$ & $14(52)$ & 0.432 & & \\
\hline \multicolumn{6}{|l|}{ Favorable outcome } \\
\hline (modified Rankin Scale <2) & $23(79)$ & $20(74)$ & 0.643 & & \\
\hline
\end{tabular}

Values are mean $\pm \mathrm{SD}, n(\%)$, or median (IQR), as appropriate. NIHSS, National Institutes of Health Stroke Scale. * Univariate analysis was performed between with and without improvement at 12 months. ${ }^{\dagger}$ Multivariable regression analysis for improvement adjusted for age, sex, current smoking, NIHSS, and ischemic stroke.

substituted for anticoagulation 6 months after onset, when the vascular lesion had shown improvement or had not changed significantly. Concurrent treatment was chosen in only 2 patients.

\section{Discussion}

This retrospective cohort of 56 consecutive Asian patients with VAD found the following: (1) the most common symptom was headache and/or posterior neck pain, with ischemic stroke occurring in only 32 cases (57\%); (2) improvement of VAD occurred in 28 cases (50\%), mainly within the initial 6 months after symptom onset; (3) older age and current smoking were independent negative predictors of improvement in VAD; and (4) aggravation of VAD was seen in 8 patients (14\%), all within 1 month of onset.

Headache and/or posterior neck pain is one of the most important clinical symptoms of VAD. The frequency of headache and/or posterior neck pain in the present series was $73 \%$ (41 of 56), comparable to other studies showing rates of $65-70 \%[7,12]$. Cerebral ischemia was found in 32 patients (57\%), lower than previous reports of $73-89 \%[1,7,12,13]$. Neuroradiological screening for VAD in patients complaining of headache has become widespread, especially in Japan [14], which may have contributed to the high incidence of VAD isolated from stroke or the low frequency of stroke.

Our study included 53 patients (94\%) with intracranial VAD, $3(4 \%)$ with extracranial VAD, and $1(2 \%)$ with both intra- and extracranial VAD, consistent with previous reports [2, 13]. One review of arterial dissections [2] reported that extracranial and internal carotid artery dissections were more common in Caucasian populations, whereas intracranial dissec- 


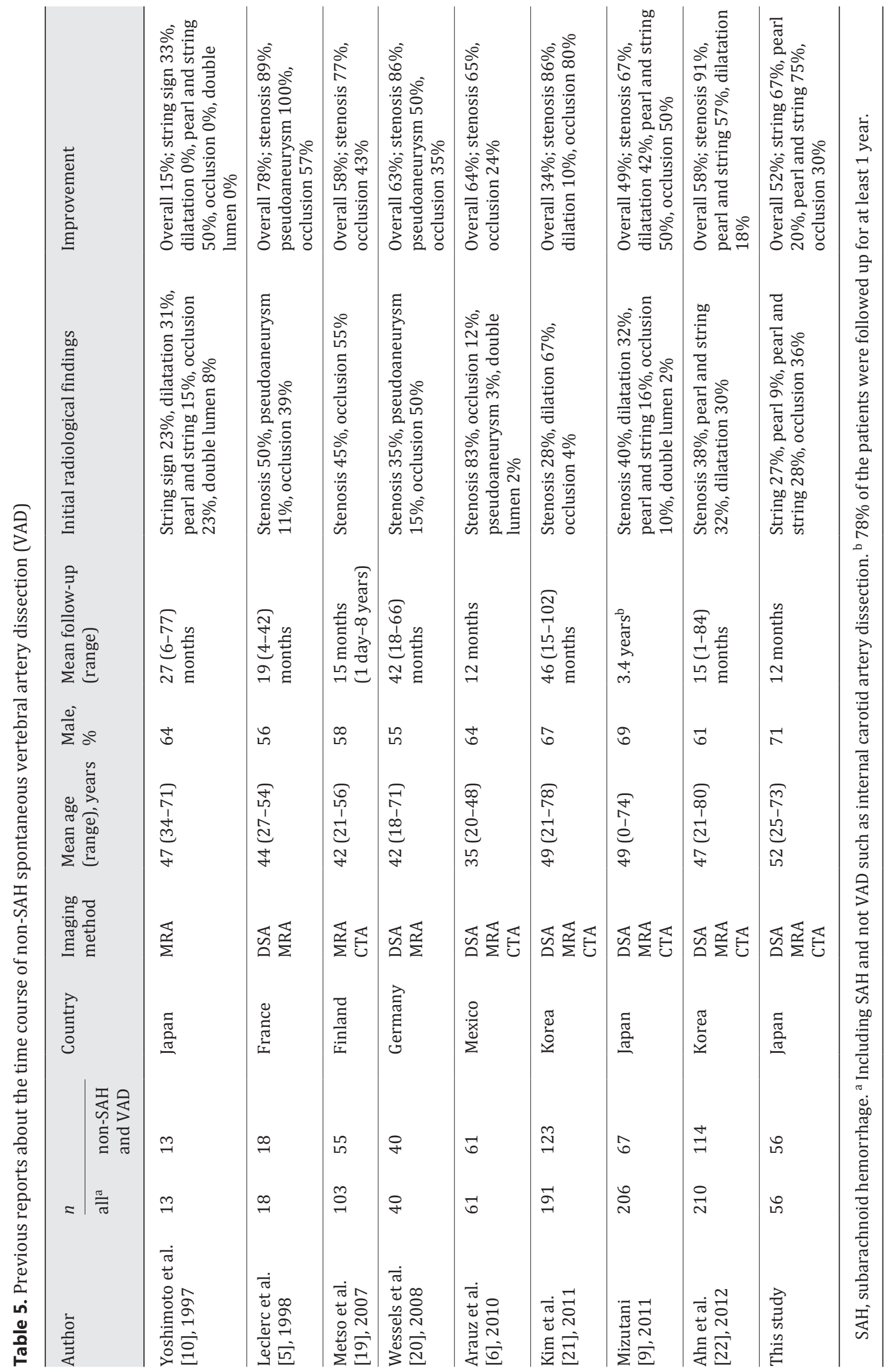


tions and VAD are more common in Asian populations. In addition, Mizutani [9] reported that the posterior circulation accounted for $88 \%$ of Japanese intracranial artery dissections.

In this study, improvement of VADs was observed mainly within the initial 6 months after symptom onset. These findings are in accordance with previous results from VAD and internal carotid artery dissection studies [6,15-17]. Recanalization is known to represent a dynamic process that may begin days or weeks after the initial dissection [18]. Hence, we propose continuing follow-up imaging for at least 6 months after onset. Arauz et al. [6] recommend maintaining antithrombotic therapy only for the first 6 months after the onset of symptoms or until complete recanalization is observed, and our findings support that proposal.

In this study, improvement of VAD occurred in 29 patients (52\%), an average figure compared with previous reports (Table 5) $[5,6,9,10,19-22]$. The pearl and string sign group appears to be more likely to show improvement. In the string sign group, a relatively high proportion of improved cases were seen, although no significant difference between with and without improvement was evident. Only 1 patient showed improvement in the pearl sign group. Some previous studies have reported that stenotic lesions tended to improve, but dilated lesions often remained unchanged [9, 22, 23]. Dissecting lesions may resolve if the neointimal hyperplasia occurring in the healing process can overcome local hemodynamic stress. However, in the case of dilated lesions, the repaired neointima may yield to the hemodynamic stress because of the extensive disruption of the internal elastic lamina [22]. On the other hand, unlike the pearl sign group, the pearl and string sign group showed aneurysm reduction in 7 of 16 patients in our study. This is because the pressure of intramural hematoma expansion towards the vascular lumen seems stronger than that towards the arterial adventitia, and thus the stenosis rather than the aneurysm is prominent in the pearl and string sign group. Hematomas are absorbed over time, so the stenosis improves and the pearl becomes less conspicuous. In the pearl sign group, intramural hematoma caused by tearing of the internal elastic lamina extends the adventitia and forms an aneurysm. The enlarged vascular adventitia might then not be restored. Our study included a small sample size, and our results thus require confirmation in a study with a large cohort.

To the best of our knowledge, this study provides the first evidence that the pearl and string sign may be more common on the dominant side of VAD, while the occlusion sign more frequently involves the nondominant side. The dissection cavity may obstruct the vascular lumen on the nondominant side more easily than the dominant side, because the diameter of the lumen is smaller on the nondominant side. Conversely, on the dominant side with a large diameter, the pearl and string sign is frequently seen because occlusion seems less frequent. In addition, pearl sites tend to be formed because blood flow on the dominant side is greater than on the nondominant side, so the pearl and string sign is more common on the dominant side of VAD.

Many previous reports have demonstrated associations between age and vascular disease. In our series, current smoking was also an independent negative predictor of VAD improvement. Arauz et al. [6] did not find any association between recanalization and cardiovascular risk factors, whereas Caso et al. [23] reported male sex and hypertension as negatively influencing recanalization. Our finding of a negative relationship between current smoking and VAD improvement is novel, which may be based on the fact that smoking history has a negative impact on the repair process of arterial dissection. In smokers, oxidative stress [13], an activated coagulation cascade [24], and endothelial dysfunction [25] might disturb VAD improvement. Furthermore, cigarettes promote inflammation in the vascular walls and blood, increases in oxidized low-density lipoprotein cholesterol, and excessive growth of smooth muscle cells, and then progression of arteriosclerosis impairs the repair process [26].

In our study, aggravation of VAD was shown in 8 patients (14\%), all within 1 month of onset. In cases of exacerbation, enlargement of the aneurysm and/or progression of stenosis 
occurred before the neointima at the dissection site was sufficiently formed. Hence, we should perform follow-up imaging to avoid overlooking aggravation of VAD, especially within 1 month after onset.

\section{Limitations}

This study has several limitations. First, we investigated the data retrospectively. Second, follow-up imaging was not standardized for all cases, but we performed MRA for all patients and DSA for almost all patients on admission. In addition, the rate of MR examination during follow-up was high. Third, this study had a small sample size. However, VAD is a rare disease, and we included a relatively large number of cases for a single-center study. Fourth, complete exclusion of atherosclerotic VA stenosis is difficult because we diagnosed VAD with luminal imaging techniques without high-resolution MRI. The high-resolution MRI is one of the most important and popular vessel wall imaging techniques for directly evaluating the vascular wall and intracranial artery disease $[27,28]$. This technique can advance the diagnostic accuracy of VAD. Finally, the choice and duration of antithrombotic therapy was not identical for all patients but was at the discretion of the treating physician, because no recommended antithrombotic treatments have been defined for VAD.

\section{Conclusions}

VAD improvement primarily occurs within 6 months after onset, and VAD aggravation within 1 month. In terms of risk factors, it seems that older patients and current smoking are negative predictors of VAD improvement, and regarding image findings, the pearl and string sign is a positive and occlusion a negative predictor.

\section{Acknowledgment}

The authors thank Kotaro Yasumori, Department of Radiology, Kyushu Medical Center, for image diagnosis of VAD.

\section{Funding Sources}

No funding was received for this study.

\section{Disclosure Statement}

The authors have no conflicts of interest to declare.

\section{References}

1 CADISS trial investigators; Markus HS, Hayter E, Levi C, Feldman A, Venables G, Norris J: Antiplatelet treatment compared with anticoagulation treatment for cervical artery dissection (CADISS): a randomised trial. Lancet Neurol 2015;14:361-367.

-2 Debette S, Compter A, Labeyrie MA, Uyttenboogaart M, Metso TM, Majersik JJ, et al: Epidemiology, pathophysiology, diagnosis, and management of intracranial artery dissection. Lancet Neurol 2015;14:640-654.

-3 Auer SF A, Schmidauer C, Waldenberger P, Aichner F: Magnetic resonance angiographic and clinical features of extracranial vertebral artery dissection. J Neurol Neurosurg Psychiatry 1998;474-481.

-4 Chen CJ, Tseng YC, Lee TH, Hsu HL, See LC: Multisection CT angiography compared with catheter angiography in diagnosing vertebral artery dissection. AJNR Am J Neuroradiol 2004;769-774. 
Shibahara et al.: Improvement of Vertebral Artery Dissection

5 Leclerc X, Lucas C, Godefroy 0, Nicol L, Moretti A, Leys D, Pruvo JP: Preliminary experience using contrastenhanced MR angiography to assess vertebral artery structure for the follow-up of suspected dissection. AJNR Am J Neuroradiol 1999;1482-1490.

-6 Arauz A, Marquez JM, Artigas C, Balderrama J, Orrego H: Recanalization of vertebral artery dissection. Stroke 2010;41:717-721.

7 von Babo M, De Marchis GM, Sarikaya H, Stapf C, Buffon F, Fischer U, Heldner MR, Gralla J, Jung S, Simonetti BG, Mattle HP, Baumgartner RW, Bousser MG, Arnold M: Differences and similarities between spontaneous dissections of the internal carotid artery and the vertebral artery. Stroke 2013;44:1537-1542.

-8 Dittrich R, Rohsbach D, Heidbreder A, Heuschmann P, Nassenstein I, Bachmann R, Ringelstein EB, Kuhlenbaumer G, Nabavi DG: Mild mechanical traumas are possible risk factors for cervical artery dissection. Cerebrovasc Dis 2007;23:275-281.

9 Mizutani T: Natural course of intracranial arterial dissections. J Neurosurg 2011;114:1037-1044.

10 Yoshimoto Y, Wakai S: Unruptured intracranial vertebral artery dissection. Clinical course and serial radiographic imagings. Stroke 1997;28:370-374.

-11 Landis JR, Koch GG: The measurement of observer agreement for categorical data. Biometrics 1977;33:159174.

12 Debette S, Metso T, Pezzini A, Abboud S, Metso A, Leys D, et al; Cervical Artery Dissection and Ischemic Stroke Patients Group: Association of vascular risk factors with cervical artery dissection and ischemic stroke in young adults. Circulation 2011;123:1537-1544.

-13 Arnold M, Kurmann R, Galimanis A, Sarikaya H, Stapf C, Gralla J, Georgiadis D, Fischer U, Mattle HP, Bousser MG, Baumgartner RW: Differences in demographic characteristics and risk factors in patients with spontaneous vertebral artery dissections with and without ischemic events. Stroke 2010;41:802-804.

14 Yamada S, Ohnishi H, Takamura Y, Takahashi K, Hayashi M, Kodama Y, Kuga Y, Nakase H, Nakagawa I: Diagnosing intra-cranial and cervical artery dissection using MRI as the initial modality. J Clin Neurosci 2016;33: 177-181.

15 Nedeltchev K, Bickel S, Arnold M, Sarikaya H, Georgiadis D, Sturzenegger M, Mattle HP, Baumgartner RW: Recanalization of spontaneous carotid artery dissection. Stroke 2009;40:499-504.

-16 Sturzenegger M, Mattle HP, Rivoir A, Baumgartner RW: Ultrasound findings in carotid artery dissection: analysis of 43 patients. Neurology 1995;45:691-698.

17 Desfontaines P, Despland PA: Dissection of the internal carotid artery: aetiology, symptomatology, clinical and neurosonological follow-up, and treatment in 60 consecutive cases. Acta Neurol Belg 1995;95:226-234.

-18 Brott T, Adams HP Jr, Olinger CP, Marler JR, Barsan WG, Biller J, Spilker J, Holleran R, Eberle R, Hertzberg V, et al: Measurements of acute cerebral infarction: a clinical examination scale. Stroke 1989;20:864-870.

19 Metso TM, Metso AJ, Helenius J, Haapaniemi E, Salonen O, Porras M, Hernesniemi J, Kaste M, Tatlisumak T: Prognosis and safety of anticoagulation in intracranial artery dissections in adults. Stroke 2007;38:18371842.

20 Wessels T, Mosso M, Krings T, Klotzsch C, Harrer JU: Extracranial and intracranial vertebral artery dissection: long-term clinical and duplex sonographic follow-up. J Clin Ultrasound 2008;36:472-479.

21 Kim BM, Kim SH, Kim DI, Shin YS, Suh SH, Kim DJ, Park SI, Park KY, Ahn SS: Outcomes and prognostic factors of intracranial unruptured vertebrobasilar artery dissection. Neurology 2011;76:1735-1741.

22 Ahn SS, Kim BM, Suh SH, Kim DJ, Kim DI, Shin YS, Ha SY, Kwon YS: Spontaneous symptomatic intracranial vertebrobasilar dissection: initial and follow-up imaging findings. Radiology 2012;264:196-202.

23 Caso V, Paciaroni M, Corea F, Hamam M, Milia P, Pelliccioli GP, Parnetti L, Gallai V: Recanalization of cervical artery dissection: influencing factors and role in neurological outcome. Cerebrovasc Dis 2004;17:93-97.

24 Mitchell GM, McCann JJ, Rogers IW, Hickey MJ, Morrison WA, O’Brien BM: A morphological study of the longterm repair process in experimentally stretched but unruptured arteries and veins. Br J Plast Surg 1996;49: 34-40.

25 Celermajer DS, Adams MR, Clarkson P, Robinson J, McCredie R, Donald A, Deanfield JE: Passive smoking and impaired endothelium-dependent arterial dilatation in healthy young adults. N Engl J Med 1996;334:150154.

-26 Unverdorben M, von Holt K, Winkelmann BR: Smoking and atherosclerotic cardiovascular disease. Part II. Role of cigarette smoking in cardiovascular disease development. Biomark Med 2009;3:617-653.

-27 Alexander MD, Yuan C, Rutman A, Tirschwell DL, Palagallo G, Gandhi D, Sekhar LN, Mossa-Basha M: Highresolution intracranial vessel wall imaging: imaging beyond the lumen. J Neurol Neurosurg Psychiatry 2016; 87:589-597.

28 Choi YJ, Jung SC, Lee DH: Smoking and atherosclerotic cardiovascular disease. Part II. Role of cigarette smoking in cardiovascular disease development. J Stroke 2015;17:238-255. 